\title{
Theoretical optimization of GalnP/GaAs dual-junction solar cell: Toward a $36 \%$ efficiency at 1000 suns
}

\author{
Mathieu Baudrit* and Carlos Algora \\ Instituto de Energía Solar, Universidad Politécnica de Madrid, Avda Complutense s/n, 28040 Madrid, Spain
}

PACS 81.05.Ea, 84.60.Bk, 84.60.Jt, 85.30.De

\begin{abstract}
A theoretical conversion efficiency of $36.4 \%$ at 1000 suns concentration has been determined by means of realistic models and an improved optimization routine. The starting point device was the recent world-record monolithic GaInP/GaAs dualjunction solar cell that was grown lattice matched on a GaAs substrate by MOVPE, which has an efficiency of $32.6 \%$ at 1000
\end{abstract}

suns. Using previously calibrated models developed at our institution, IES-UPM, together with Silvaco ATLAS TCAD software, we reproduced the characteristics of the world-record solar cell, and then determined a cell configuration that would yield greater efficiency by using an optimization routine to hone the doping concentration and the thickness of each layer.
1 Introduction Since 1960s, when solar-cell concepts really flourished, new structural and material concepts have emerged to lead us toward higher-efficiency and lower-cost devices. Between the high-efficiency concepts, multijunction solar cells (MJC) have overcome the $40 \%$ barrier with record efficiencies of up to $41.4 \%$ [1] at 454 suns using triple-junction devices and yield a $32.6 \%$ efficiency at 1000 suns using dual-junction devices [2]. When deployed with solar-concentrator systems these devices have become a true contender in the marketplace. Notwithstanding this, the very high efficiencies achieved by these devices is due to their great complexity, which make their experimental optimization complicated, time consuming, and expensive. This is where numerical modeling appears to be a valuable tool that allows automated solar-cell-design optimization, decreasing costs and development time. Some reliable modeling results have already been obtained for III-V single-junction solar cells using commercially available semiconductor simulation tools [3-5]. To obtain such good results it is important to take into account a wide range of effects occurring inside these cells, such as optical interference effects, carrier transport at heterointerfaces, trap effects, and so on. Another aggravating factor in the case of MJC consists in the fact that two or more subcells need to be computed simultaneously. These subcells are connected via Esaki tunnel diodes whose models have to be included in the simulation in order to acquire a better understanding of the effects limiting cell efficiency. For this, a suitable and numerically stable nonlocal model for the tunnel diodes is essential. Dual-junction solar cells have recently been successfully modeled $[6,7]$. In one of these studies, initial attempts were made to optimize the emitter and base of dualjunction solar cells [8]. Therefore, a wider optimization of the dual-junction solar cell seems to be necessary in order to reach the efficiency limit of the practical device. Accordingly, the aim of the present work is to optimize as much as possible the whole semiconductor structure of the dualjunction solar cell. In our case we used different software, namely Silvaco ATLAS. We show that this software can be coupled to a routine that allows complete automation of the optimization process under certain restricting conditions fixed by the user. So, the focus of this paper is the optimization process and the results obtained. The detailed layer structure is treated as proprietary for both the starting point device and the optimized device, and hence is not reported here.

2 Description of the studied solar cell The structure studied in this paper consists of a lattice-matched dual-junction $\mathrm{GaInP} / \mathrm{GaAs}$ solar cell. The device was grown by MOVPE and a cross-section of its structure is depicted in Fig. 1. The contact layer under the metallization grid is 


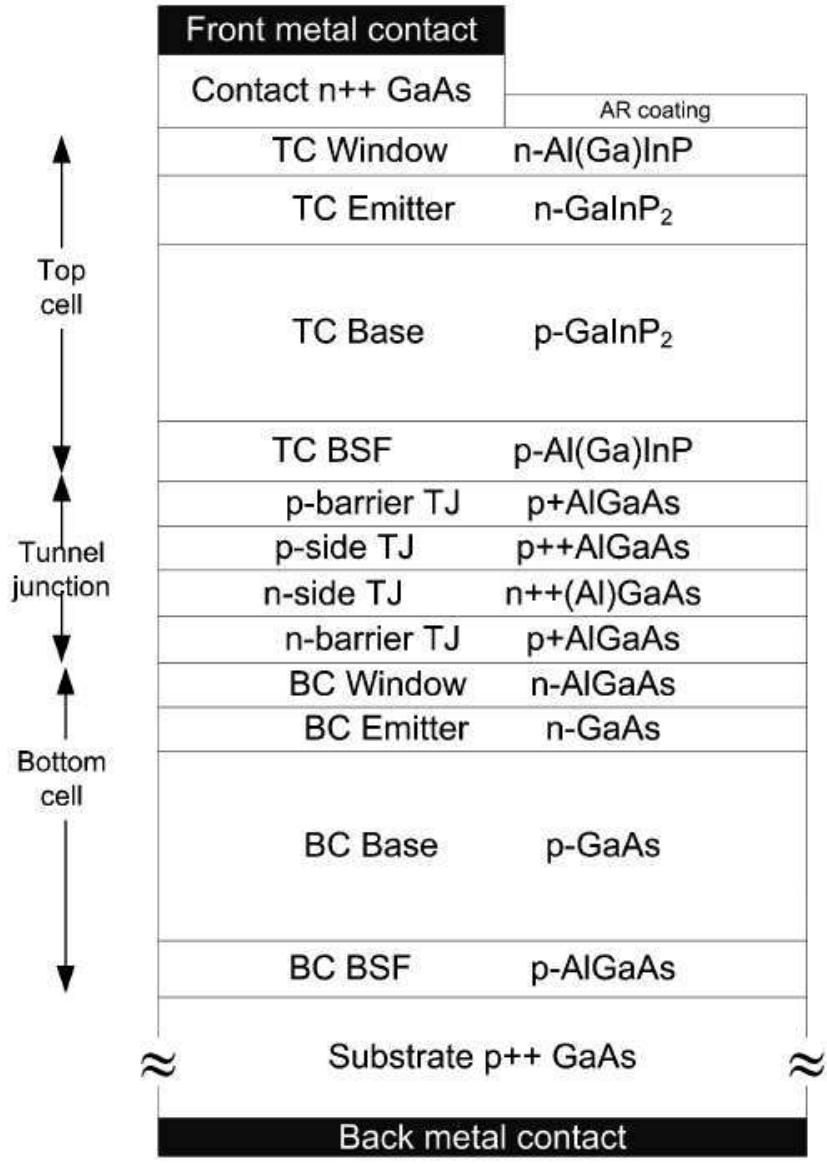

Figure 1 Semiconductor structure of the GaInP/GaAs dualjunction solar cell modeled and optimized in this work.

formed by GaAs. The tunnel diode consists of the tunnel junction itself made by two degenerately doped $n^{++}$-GaAs and $\mathrm{p}^{++}$-AlGaAs layers as well as two AlGaAs enclosing barrier layers with the purpose to minimize dopant diffusion. The structure was processed by using photolithography techniques to define the front grid, and the metal was deposited by means of thermal evaporation. The wet mesa etching process applied to isolate the devices and minimize the perimeter recombination consisted of an optimized sequence of etching processes using acid and basic solutions. A triple layer $\mathrm{MgF} / \mathrm{ZnS} / \mathrm{MgF}$ antireflective coating (ARC) was finally deposited on the device, which was encapsulated. Additional details about the structure can be found in Ref. [9]. This device recently yielded the world-record efficiency of $32.6 \%$ at 1000 suns [2]. This is of great interest for the purpose of this paper as we will show that optimization of the automation routine allows fast and significant improvement of a structure whose efficiency is already high.

3 Overview of the simulation environment The numerical simulation of semiconductor processes and devices, often referred to as technology computer-aided design (TCAD), is a well-established discipline in the world of semiconductor process development and optimization currently used by many semiconductor laboratories and companies in applications ranging from nanoscale CMOS to power devices and RF semiconductor devices. In the case of solar cells, the use of TCAD is quite recent and connected to the increased complexity of high-efficiency structures and to the amount of detailed phenomena that have to be taken into account, needing then more powerful tools to reproduce their behavior and correctly model the physics involved. Device simulation comprises the solution of semiconductor transport equations within semiconductor regions with external metal contacts and is broadly applied to Si-based devices but can also be extended to compound semiconductors as materials can be completely defined by the user, using ab initio, empirical or semiempirical models, and material properties.

Solar cells are electro-optical devices. For this, from the optics point of view, the reflection and transmission of the light must be treated at interfaces between media. In this case, if the thicknesses of the layers are larger than the wavelength of the light, a ray-tracing model is sufficient. When interference effects are important, such as the presence of an ARC or when semiconductor absorption layers are thin, a transfer matrix method (TMM) should be used if the structure is planar or quasiplanar, as in our case. From the electric point of view, it is important to include the changes in energy gap caused by heavy doping effects and composition. Radiative, Auger and Shockley-Read-Hall (SRH) recombination are also very important because any process removing carriers from collection by the cell terminals ultimately affects the efficiency. In our case, all material parameters are based on empirical models (from the literature or developed at IES-UPM $[10,11])$ verified by measurements to reach reliable and realistic models corresponding to the materials grown in our laboratory.

A special emphasis was also made in the tunnel diode modeling. In normal condition of operation, it could be easily replaced by a resistance to carry out the simulation. With the fault that this simplification does not reproduce the absorption of light inside the tunnel diode, especially important in the case of device studied herein, where the AlGaAs/GaAs tunnel junction absorbs a considerable quantity of light as compared to other high bandgap systems. This workaround is also unable to reproduce the failure of the MJC due to tunnel diode when the solar-cell short-circuit current $J_{\mathrm{sc}}$ is higher than the peak current admitted by the tunnel junction. Therefore, we have included a tunnel diode model that fits completely the entire $I-V$ curve as shown in Ref. [6].

4 Model verification In our simulation, a twodimensional (2D) symmetry element of the cell is modeled; hence, series-resistance effects due to lateral and vertical current flows are also taken into account as carrier dynamics calculation is made in 2D. After successfully simulating single-junction solar cells, the tunnel junction was also included using a complete nonlocal tunneling model based on the WKB tunneling probability taking into account local 


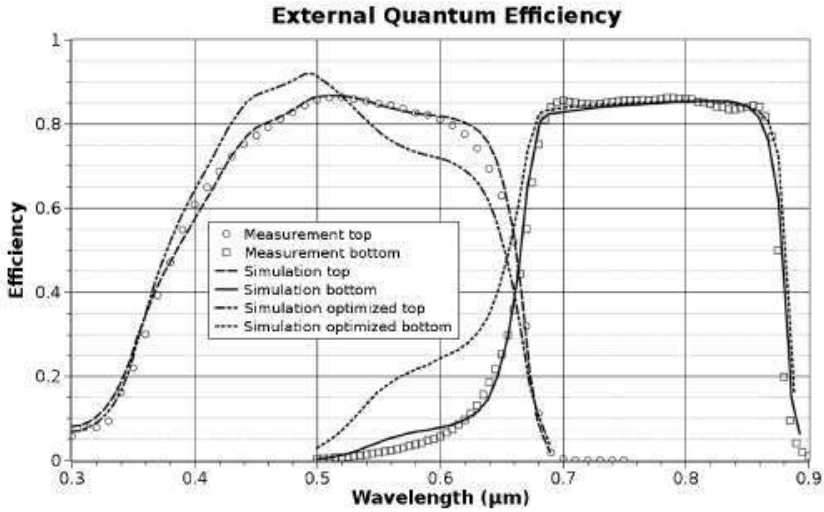

Figure 2 Measured external quantum efficiency (dots), simulated external quantum efficiency before and after optimization.

and nonlocal traps effects. This model has previously shown very good results in simulations of tunnel diode and dualjunction solar cells [6], despite a small mismatch implied in the fill factor prediction when modeling the solar-cell $I-V$ curve. These latter problems have been solved recently and we now have a complete and reliable model for dual-junction solar cells. To verify the models employed, the first simulation was performed to determine the external quantum efficiency. The results are shown in Fig. 2. A fairly good fit can be observed between the experimental and simulation data. The $J_{\mathrm{sc}}$ at 1 sun calculated by convolution of the measured EQE of the ARC-coated devices with the standard solar spectrum AM1.5D low-AOD shows a value of 14.3 and $13.5 \mathrm{~mA} / \mathrm{cm}^{2}$ for the top and bottom cell, respectively, indicating that there exists a current mismatch of $6 \%$ and that the efficiency can still be improved. Next, we proceed with simulating the $I-V$ curve. The results, detailed in Fig. 3, show that the model match perfectly the device behavior, allowing us to extract the $J_{\mathrm{sc}}, V_{\mathrm{oc}}, \mathrm{FF}$, and efficiency at 1026 suns, the concentration used to measure the world-record dualjunction solar cell. These results confirm the validity of the tunneling model over the whole current and voltage range (not only at zero bias as in the EQE) and reassert the capacity of the TCAD tools developed for the optimization process.

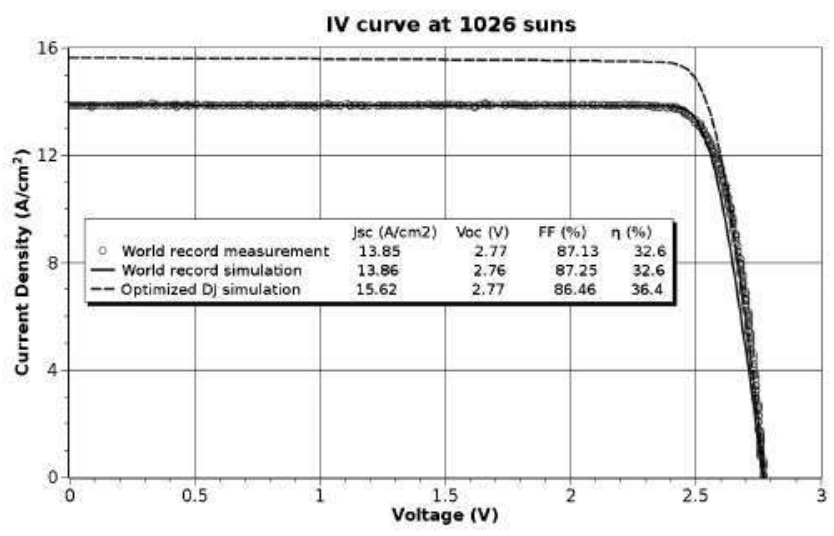

Figure $3 l-V$ curve of the studied solar cell under AM1.5D lowAOD spectrum at 1026 suns; measured (dots), simulated (solid line), and simulated after optimization (dash line).
5 Optimization The simplest method to optimize a structure in terms of efficiency is a so-called step-by-step optimization process, which consists of optimizing each layer thickness and doping concentration at once. In this case, if we wish to optimize the window, emitter, base, and BSF of both the top cell and bottom cell, we have a total of 8 layers, and hence a total of 16 variables ( 2 per layer: the thickness and the doping concentration). If we perform eight simulations per variable in a two-pass process - the first pass to determine the approximate best value for each variable and then the second pass to refine the results - we will need a total of 256 runs. This optimization method is probably the easiest to implement in simulation software like Silvaco ATLAS. However, this method is quite weak because the variables to be optimized are treated as separate when in reality they are linked. An example of this fault is that even using a three- or four-pass process, the maximum efficiency achieved is of $34 \%$ and vary depending on which variable we optimize first, showing then that the best value for each variable depends on the values of the others and that this method is not reliable enough. Furthermore, the step-by-step method works with a fixed number of runs that depends on the number of variables to be optimized.

As an alternative to the step-by-step optimization process, a crossed variable optimization process can be implemented. In our case, we used a modified LevenbergMarquardt (LM) algorithm [12-15]. The LM algorithm is an iterative technique that locates the minimum of a multivariable function that is expressed as the sum of squares of nonlinear real-valued functions. LM can be thought of as a combination of steepest-descent and the Gauss-Newton method. When the current solution is far from the correct one, the algorithm behaves like a steepest-descent method: slow, but guaranteed to converge. When the solution is close to the correct solution, it becomes a Gauss-Newton method. In this case, the number of runs is not fixed beforehand; the algorithm determines the influence of each variable on the target results (the efficiency at 1026 suns in our case) and then proceeds to trace the most efficient way to maximize the target, considering the complex interactions among the variables.

For the aim of this paper we worked with a tuned version of LM. It was modified to search for a local maximum in the efficiency rather than the absolute maximum efficiency. Our method seeks an efficiency resulting from a compromise between stability and maximization, in order to achieve values that can be easily transferred to technological processes with reasonable margins of error. These margins of error are defined in Table 1 along the minimum and maximum values allowed for each variable, as said before, the nominal values are treated as proprietary and will not be revealed.

Hence, the condition fixed to the optimizer was to find layers parameters whose variation inside the margin of error does not affect the final efficiency by more than an absolute $0.2 \%$. This modification is very important because in many systems the configuration with the highest efficiency suffers 
Table 1 List of variables introduced into the optimizer along with their minimum, maximum, and technological margins of error.

\begin{tabular}{llllll}
\hline subcell & variable & unit & minimum & maximum & technological margin \\
\hline top cell & window thickness & $\mu \mathrm{m}$ & 0.0125 & 0.048 & \pm 0.005 \\
& window doping & $\mathrm{cm}^{-3}$ & $3 \times 10^{16}$ & $5 \times 10^{17}$ & $\pm 1 \times 10^{16}$ \\
& emitter thickness & $\mu \mathrm{m}$ & 0.065 & 0.265 & $\pm 10 \%$ \\
& emitter doping & $\mathrm{cm}^{-3}$ & $1 \times 10^{17}$ & $1 \times 10^{19}$ & $\pm 1 \times 10^{17}$ \\
& base thickness & $\mu \mathrm{m}$ & 0.215 & 0.9 & $\pm 10 \%$ \\
& base doping & $\mathrm{cm}^{-3}$ & $1 \times 10^{15}$ & $1 \times 10^{17}$ & $\pm 1 \times 10^{16}$ \\
& BSF thickness & $\mu \mathrm{m}^{-3}$ & 0.025 & $0.110^{16}$ & $\pm 10 \%$ \\
& BSF doping & $\mathrm{cm}^{-3}$ & $7 \times 10^{16}$ & $7 \times 10^{18}$ & $\pm 1 \times 10^{16}$ \\
bottom cell & window thickness & $\mu \mathrm{m}^{-3}$ & 0.01 & 0.045 & \pm 0.005 \\
& window doping & $\mathrm{cm}^{-3}$ & $6 \times 10^{16}$ & $6 \times 10^{18}$ & $\pm 1 \times 10^{16}$ \\
& emitter thickness & $\mu \mathrm{m}^{-3}$ & 0.04 & 0.155 & $\pm 10 \%$ \\
& emitter doping & $\mathrm{cm}^{-3}$ & $6 \times 10^{16}$ & $6 \times 10^{18}$ & $\pm 1 \times 10^{16}$ \\
& base thickness & $\mu \mathrm{m}^{-3}$ & 1.5 & 5.5 & $\pm 10 \%$ \\
& base doping & $\mathrm{cm}^{-3}$ & $3 \times 10^{15}$ & $3 \times 10^{17}$ & $\pm 0.5 \times 10^{16}$ \\
BSF thickness & $\mu \mathrm{m}^{-3}$ & 0.06 & 0.25 & $\pm 10 \%$ \\
& BSF doping & $\mathrm{cm}^{-3}$ & $1 \times 10^{17}$ & $1 \times 10^{19}$ & $\pm 0.5 \times 10^{18}$ \\
\hline
\end{tabular}

from the shortcoming that a small variation in one of the layer characteristics translates into a large drop in efficiency. leading to strong variations in the results obtained. Table 1 also shows how some variables ranges were shortened, defining a minimum and maximum authorized values, like the top cell window doping concentration for example, as we know it is difficult to dope AllnP material to values higher than $5 \times 10^{17} \mathrm{~cm}^{-3}$. This control over the possible values have been introduced in an attempt to achieve realistic optimized values as an optimization is meaningless if it is impossible to transfer to technology.

Concerning the assumptions made during the optimization. we considered the metallization grid (contact size and spacing) along with its specific contact resistance, the surface recombination velocity between layers, the tunnel junction structure, and the material quality as constants. All the top cell and bottom cell structures were considered in the optimization process, including the window and BSF layers. These layers are often seen as merely technological layers whose characteristics have little influence on the device performance; however. these layers influence the band diagram of the whole structure and also contribute to photogeneration and, for these reasons, should be taken into account to achieve a realistic and complete optimization. When applied to our structure, the LM optimization method required 23 runs $(9 \mathrm{~h}$ on a bi-dual-core processor machine) to calculate the best structure for the AM1.5D lowAOD spectrum at a concentration of 1026 suns, much faster than the step-by-step method, which requires 256 runs to achieve a worse result. Figure 3 summarizes the results obtained before and after the crossed variable optimization process.

6 Results As can be seen in Fig. 3, the optimization strongly increased the current density of the device from 13.85 to $15.62 \mathrm{~A} / \mathrm{cm}^{2}$ at 1026 suns, without greatly changing the $V_{o c}$ but reducing slightly the FF to $86 \%$, leading to an efficiency of $36.4 \%$ at this concentration. This variation in $\mathrm{FF}$ is logical as the original device features an increased FF due to the current mismatch but the loss in short-circuit current produces a net conversion efficiency that is lower than that in a current-matched device.

This efficiency obtained corresponds to the local maximum allowing reasonable technological margins of error. and is slightly below the absolute maximum calculated. This effect on $J_{\mathrm{sc}}$ can be appreciated in the simulated external quantum efficiency where the previous current mismatch completely disappeared. We found that the optimization, which focused on maximizing efficiency. mainly increases the response of the top cell to the shortest wavelengths, while increasing the bottom cell current and suppressing the current mismatch. However, the top cell blue response is still poor, as in the original device, as a consequence of the emitter design, which is optimized to maximize the efficiency at 1000 suns by reducing seriesresistance component. This leads to the necessity of using a thickness and doping level in this layer that produce such an EQE response.

To reach this high current density eliminating the current mismatch, the optimizer worked actively on the characteristics of the window, emitter, base, and BSF of both subcells. In the case of the top cell, the window has been made thicker with a higher doping concentration, joined with a higherdoped emitter, resulting then in a better response of the top cell to the shortest wavelength. However, the emitter and base have been made thinner to enhance the photogeneration rate of the bottom cell avoiding to lead to a bottom cell limited dual-junction solar cell. In the case of the bottom cell, the window layer has been made much thinner to have a better response of this cell to the shortest wavelength. The emitter and base strongly increased their thicknesses to reach higher values of the current density. The stability of the efficiency has also to be noted. Indeed, a 10\% variation of the value of the thickness and doping concentration only affect 
the resulting efficiency by an absolute increase (or decrease) of $0.05 \%$. allowing very good technological margins of error.

7 Conclusion The current world-record efficiency for dual-junction solar cells at 1000 suns is $32.6 \%$. We introduced the complete structure of this device into Silvaco software and. using models developed at IES-UPM, achieved a good match between simulation and measurements in the case of the external quantum efficiency and $I-V$ curve under illumination. After verifying the accuracy of the models employed, we introduced the parameters of this cell into an optimization routine that succeeded in increasing the efficiency at 1026 suns by $4 \%$ (absolute), leading to a theoretical but achievable efficiency of $36.4 \%$. Our approach of using an adapted optimization routine and a set of realistic models opens new possibilities for experimental/theoretical feedback in order to achieve higher efficiencies in a very short time.

Acknowledgements The authors are deeply indebted to C. Tablero for useful discussions on simulation and optimization, the other members of the III-V semiconductors group, and to Dr. A. Bett and G. Sieffer from the FhG ISE for the certified EQE and concentration measurements. Funding for this research was provided by the European Commission under Contract No. SES6-CT-2003-502620 (FULLSPECTRUM project), by the Spanish Ministry of Education and Science (Ministerio de Educación y Ciencia) through projects GENESIS-FV (part of the CONSOLIDER-INGENIO 2010 program), Contract Nos. TEC2005-02745 and TEC2008-01226, and by the Regional Government of Madrid (Comunidad de Madrid) under Contract No. S-505/ENE/0310 (NUMANCIA program).

\section{References}

[1] FhG press release, www.ise.fraunhofer.de.

[2] C. Algora, L. Rey-Stolle, I. García, B. Galiana, M. Baudrit, P. Espinet, E. Barrigón, A. Datas, J. R. González, and J. Bautista, A dual junction solar cell with an efficiency of $32.6 \%$ at $1000 \mathrm{X}$ and $31.0 \%$ at $3000 \mathrm{X}, 5$ th ICSC, Palm Desert (USA), 2008.

[3] M. Baudrit and C. Algora, 3D modeling of concentrator III-V multi-junction solar cells, 2006 IEEE 4th WCPEC. Vols. 1 and 2, 2006.

[4] M. Baudrit and C. Algora, Numerical analysis of GaInP solar cells: Toward advanced photovoltaic devices modeling, Proceedings of the 5th NUSOD, Berlin, Germany, 2005, pp. 41-42.

[5] G. Letay, M. Hermle, and A. W. Bett, Prog. Photovolt. Res. Appl. 14, 683-696 (2006).

[6] M. Baudrit and C. Algora, in: Modeling of GaInP/GaAs DualJunction Solar Cells including Tunnel Junction, Photovolatic Specialists Conference (PVSC). 33rd IEEE 11-16 May 2008, pp. $1-5$.

[7] M. Hermle, G. Letay, S. P. Philipps, and A. W. Bett, Prog. Photovolt. Res. Appl. 16, 409-418 (2008).

[8] S. P. Philipps, M. Hermle, G. Letay, F. Dimroth, B. M. George, and A. W. Bett, Phys. Status Solidi RRL 2(4), 166-168 (2008).

[9] I. Garcia, I. Rey-Stolle, B. Galiana, and C. Algora, Appl. Phys. Lett. 94, 053509 (2009).

[10] S. Adachi, Properties of Group-IV, III-V and II-VI Semiconductors. Wiley Series in Materials for Electronic \& Optoelectronic Applications (Wiley, Chichester, 2009), ISBN: 978-0-470-09033-6.

[11] Atlas Users' Manual, Silvaco, 2009.

[12] C. T. Kelley, Iterative Methods for Optimization (SIAM Press, Philadelphia, 1999).

[13] K. Levenberg. Quart. Appl. Math. 2(2). 164-168 (1944).

[14] D. W. Marquardt, SIAM J. Appl. Math. 11(2), 43l-44l (1963).

[15] J. Nocedal and S. J. Wright, Numerical Optimization (Springer, New York, 1999). 